\title{
Retroperitoneal Fibrosis Presenting With Panhypopituitarism
}

M Talla ${ }^{1}$, K Hughes ${ }^{1}$, L McGeoch ${ }^{2}$, J Boyle ${ }^{1}$, R Drummond ${ }^{1}$, D Carty ${ }^{1}$

Department of Diabetes \& Endocrinology, Glasgow Royal Infirmary ${ }^{1}$

Department of Rheumatology, Glasgow Royal Infirmary ${ }^{2}$

\section{Background}

A 68 year old gentleman with hypertension and diet-controlled Type 2 diabetes mellitus presented in September 2015 with weight loss, fatigue, low libido and cold intolerance.

\section{Blood Results}

\begin{tabular}{lll}
\hline & Result & Reference Range \\
\hline TSH & $0.59 \mathrm{mU} / \mathrm{L}$ & $0.35-5.00$ \\
\hline Free T4 & $8.3 \mathrm{pmol} / \mathrm{L}$ & $9.0-21.0$ \\
\hline Testosterone & $1.0 \mathrm{nmol} / \mathrm{L}$ & $10.0-36.0$ \\
\hline Sex Hormone Binding & $120 \mathrm{nmol} / \mathrm{L}$ & $13-70$ \\
Globulin & & \\
Free Testosterone & $7 \mathrm{pmol} / \mathrm{L}$ & $>200$ \\
\hline Prolactin & $795 \mathrm{mU} / \mathrm{L}$ & $<400$ \\
\hline LH & $1.1 \mathrm{U} / \mathrm{L}$ & $1.0-12.0$ \\
\hline FSH & $1.5 \mathrm{U} / \mathrm{L}$ & $1.0-12.0$ \\
\hline Baseline cortisol & $87 \mathrm{nmol} / \mathrm{L}$ & \\
Cortisol 30 minutes post- & $376 \mathrm{nmol} / \mathrm{L}$ & \\
Synacthen & & \\
IGF1 & $84 \mathrm{ug} / \mathrm{L}$ & $39-186$ \\
\hline Angiotensin converting & $44 \mathrm{U} / \mathrm{L}$ & $<88$ \\
enzyme & & \\
Ferritin & $172 \mathrm{ug} / \mathrm{L}$ & $20-300$ \\
\hline IgG1 subclass & $3.68 \mathrm{~g} / \mathrm{L}$ & $3.2-10.2$ \\
\hline IgG2 subclass & $2.94 \mathrm{~g} / \mathrm{L}$ & $1.2-6.6$ \\
\hline IgG3 subclass & $1.67 \mathrm{~g} / \mathrm{L}$ & $0.2-1.9$ \\
\hline IgG4 subclass & $0.10 \mathrm{~g} / \mathrm{L}$ & $0.0-1.3$ \\
\hline
\end{tabular}

\section{Imaging}

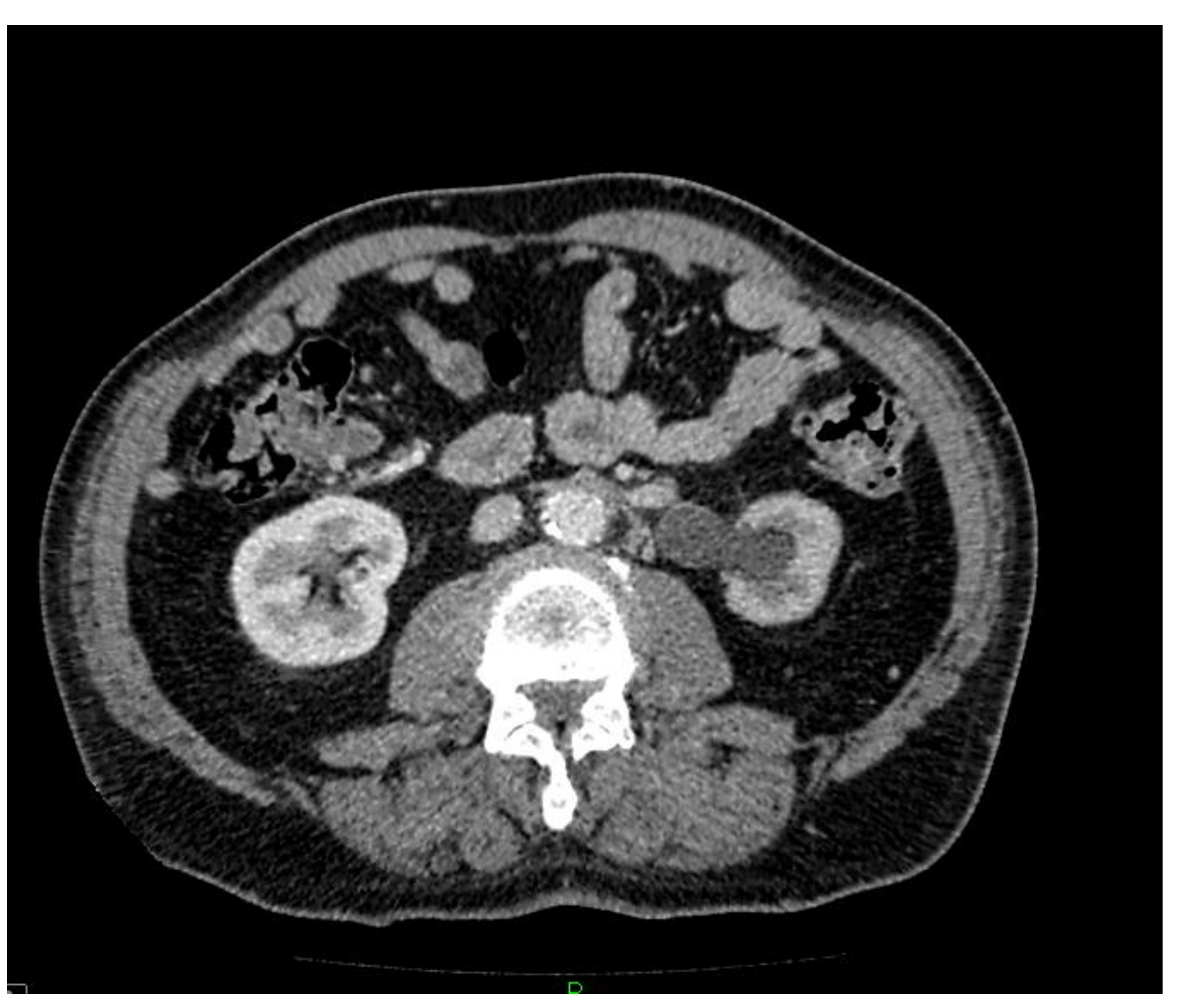

Image 1a: CT demonstrating inflammatory material extending from upper part of the left pelvic sidewall, involving iliac vessels and left ureter, resulting in left hydroureteronephrosis.

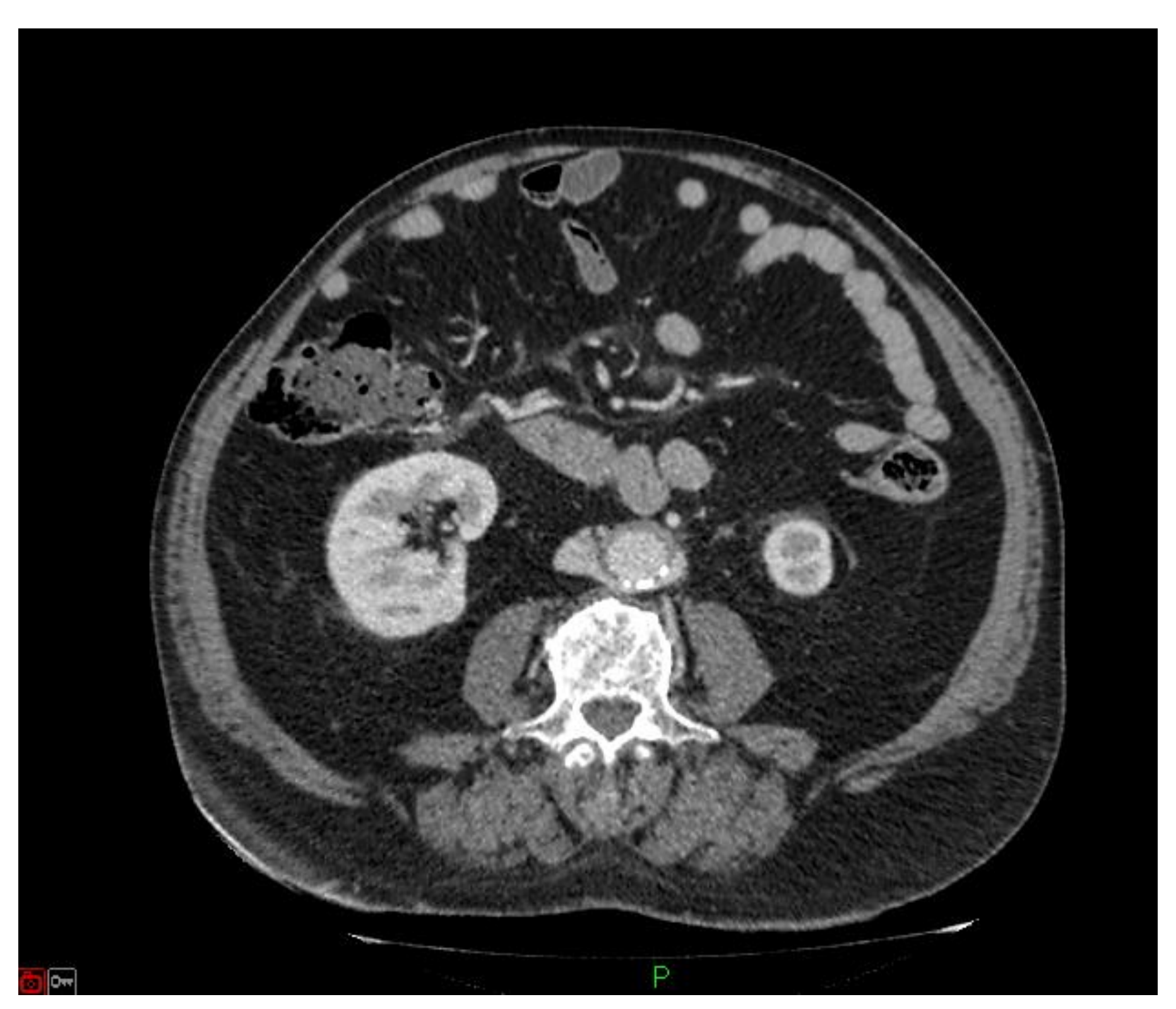

Image 2: Repeat CT performed 10 months later demonstrating a modest reduction in the inflammatory material.

Image 1b: CT demonstrating inflammatory material extending from upper part of the left pelvic sidewall, involving iliac vessels and left ureter, resulting in left hydroureteronephrosis.

\section{Clinical Progress}

Blood results demonstrated secondary hypothyroidism, hypogonadotrophic hypogonadism and a modestly elevated prolactin. Short synacthen test revealed a baseline cortisol of $87 \mathrm{nmol} / \mathrm{L}$ rising to $376 \mathrm{nmol} / \mathrm{L}$

MRI of the pituitary was carried out. This showed normal appearances of the brain and pituitary gland.

The working diagnosis was that of panhypopituitarism of unclear aetiology. He was commenced on oral hydrocortisone, levothyroxine and topical testosterone replacement therapy. This led to symptomatic improvement.

Six weeks later, he presented once again with a swollen left leg. Doppler ultrasound of the left leg at that time excluded a DVT.

In view of the the concern of potential underlying malignancy, a CT of the thorax, abdomen and pelvis was carried out. This confirmed presence of extensive inflammatory-looking material within the abdomen and pelvis, involving the iliac vessels on the left, resulting in a deep vein thrombosis at that site. The left ureter was also involved, resulting in hydroureteronephrosis.

Radiological appearances and subsequent core biopsy was in keeping with retroperitoneal fibrosis.

He was commenced on high dose oral prednisolone. Repeat imaging has since demonstrated a modest reduction in the inflammatory material around the left ureter, distal aorta and iliac vessels. He remains well and continues on prednisolone at a dose of $5 \mathrm{mg}$ once daily.

Despite the improvement in his CT appearances with corticosteroid therapy, the retroperitoneal fibrosis could progress further. Future therapeutic options would include azathioprine or methotrexate.

From a urological perspective, he has since undergone bilateral ureteric stenting due to concerns regarding future progression of the retroperitoneal fibrosis and the need to conserve his right-sided renal function.

\section{Discussion}

Immunoglobulin G4-related disease is a collection of disorders characterised by tissue infiltration with IgG4 positive plasma cells and CD4+ T lymphocytes. These features are often accompanied by fibrosis. It may affect one or more organs, and in this case manifests as a combination of retroperitoneal fibrosis and hypophysitis.

Lymphadenopathy is often present, alongside weight loss in those with multiorgan disease. It most often occurs in middle-aged men. Retroperitoneal fibrosis is a common presentation of IgG4-related disease and is likely to involve iliac vessels and ureters, leading to obstructive uropathy.

The diagnosis of IgG4-related disease is based on characteristic histopathological features on biopsy. Serum levels of IgG4 may be elevated in approximately $60-70 \%$ of patients, with the remainder having normal lgG4 levels.
Most patients respond well to corticosteroid therapy. 